\title{
Experience and perspectives of using active noise and vibration control for reduction of low frequency noise and vibration in gas guide systems of power plants
}

\author{
Andrey V. Vasilyev \\ Povolzhsky Resource Center of Engineering Ecology and Chemical Technology, Samara State Technical \\ University, Samara, Russian Federation \\ E-mail: avassil62@mail.ru \\ Received 25 May 2020; accepted 12 June 2020 \\ DOI https://doi.org/10.21595/vp.2020.21491 \\ Check for updates \\ Copyright (C) 2020 Andrey V. Vasilyev. This is an open access article distributed under the Creative Commons Attribution License, which \\ permits unrestricted use, distribution, and reproduction in any medium, provided the original work is properly cited.
}

\begin{abstract}
One of the main reasons of generation of intensive noise and vibration in low frequency range are gas pressure pulsations (oscillations) in gas guide systems of power plants. Active noise and vibration control now is considered as efficient method of low frequency noise and vibration for different applications, including reduction of low frequency noise and vibration in gas guide systems of power plants. Results of analysis of application of active noise and vibration control systems for the reduction of power plants low frequency noise and vibration are described. The examples are showing high possibilities and efficiency of active noise and vibration control method. In a meantime, existing constructions of ANVC systems are having some drawbacks. Author's experience of development of constructions and technical solutions of ANVC systems for reduction of low frequency noise and vibration in power plants gas guide systems is presented. Further perspectives of increasing of efficiency of ANVC control of low frequency noise and vibration reduction generated by power plants are discussed.
\end{abstract}

Keywords: power plant, low frequency, noise, vibration, active control.

\section{Introduction}

Low frequency noise is spreading for a long distances without significant absorption. Low frequency vibration may cause professional illnesses of workers and workers disease, negative impact to environment and to the health of inhabitant on the territory near to industrial enterprises.

Industrial and transport noise and vibration may cause professional illnesses of workers and workers disease, negative impact to environment and to the health of inhabitant on the territory near to industrial enterprises and transport networks. Intensive noise and vibration generated by power plants noise are the reason of labor safety, productivity and quality problems [1-11]. One of the main reasons of generation of intensive noise and vibration in low frequency range are gas pressure pulsations (oscillations) in gas guide systems of power plants.

Active noise and vibration control now is considered as efficient method of low frequency noise and vibration for different applications, including reduction of low frequency noise and vibration in gas guide systems of power plants.

This paper is devoted to discussion of experience and perspectives of using of active noise and vibration control for reduction of low frequency noise and vibration in gas guide systems of power plants.

\section{Analysis of application of active noise and vibration control systems for the reduction of power plants low frequency noise and vibration}

Active noise and vibration control systems may be subdivided to feedback, feed-forward and combined feedback-feedforward [1]. Feedback control systems are better to use for noise reduction of unsteady systems, whereas feedforward systems are preferable to use for steady noise cancellation. All feedback systems are close-loop. Active vibration isolation is in fact also 
close-loop.

Open loop control system historically has been developed as noise and vibration reduction in narrow band range frequency ranges and are appropriate mostly for periodical noise and vibration cancellation where reference signal may be defined. This is feedforward control.

There are also combined feedforward-feedback control systems using for increasing of efficiency of noise and vibration cancellation.

Due to the recent achievements in the field of active noise and vibration control systems the is increasing of practical application of ANVC systems. It is brightly illustrated by a huge and continuously increasing number of patents in active noise and vibration control. Presently the leading automobile companies ("General Motors", "Ford", "Lotus Engineering", "Volkswagen", "BMW", "AUDI", "Peugeot", "Nissan", etc.), consulting and research companies ("Sound Attenuators Ltd.", "Nelson Industries", "Topexpress Ltd", "Noise Cancellation Technologies, Inc.", "Active Noise and Vibration Technologies, Inc" etc.), universities, companies and research organizations (Müller BBM GmbH (Germany), Fraunhofer Institute of Building Physics (Germany), Samara State Technical University (Russia) etc., have developed a numerous number of active silencers constructions for active noise and vibration control in ducts.

The widest application of ANVC technology is active noise and vibration control of external noise generated by ICE intake and exhaust systems and of internal noise and vibration inside of transport passenger compartment. E.g. "Lotus Engineering" together with University Southampton (Great Britain) were achieved significant results in internal car noise attenuation. For different modifications of "Esprit Turbo" $10 \mathrm{~dB}$ reduction in every place of saloon have been achieved. There are also good results for some other cars. In particular, active system in "Toyota-Celica" car allows to reduce internal noise on 10-15 dB. During several years in Japan Nissan "Bluebird" car have been sold with additional system of active noise control. "McDonnell Douglas" company (USA) have presented full-scale tests of active vibration and structural noise control in DC-9 aircraft cabin. T. Berge have described active noise control system in truck cabin. For fundamental frequency $30 \mathrm{~Hz}$ reduction $15,7 \mathrm{~dB}$ have been achieved.

There are a lot of examples of successful application of ANVC technique is active noise and vibration control in automobile internal combustion engines gas-guide systems, e.g. in intake and exhaust ducts of ICE, in transport pipelines of power plants (compressors, pumps etc.). Electronic system of "Lotus" company is shown in Fig. 1.

Apparatus to attenuate dynamic pressure fluctuations of a gas flowing in a duct developed by "Lotus" company [4] comprises a valve mounted in the duct controllable to vary the cross-sectional area of the duct through which the gas can flow, sensor means to provide an indication of the dynamic pressure fluctuations to be attenuated, and control means to control the valve in response to signals from the sensor means such that the amplitude and phase of the dynamic pressure fluctuations are controlled to provide attenuation.

A separate and well-studied field is active vibration control (AVC), especially for automobiles. AVC may be used for vibrations reduction of engine body, pan, steering wheel, seats etc., see e.g. developments of "Lotus Engineering", "Carl Freudenberg" etc. E.g. R.A. Smith and G.B.B. Chaplin were developed AVC system for vibration reduction in 12-cylinder diesel engine mounts. For discrete frequencies vibration reduction achieved up to $40 \mathrm{~dB}$.

In Fraunhofer Institute of Building Physics (Germany) a lot of theoretical and experimental investigations were undertaken for the new ANC systems constructions development for the different fields of application [3, 5, etc.]. In paper [3] active absorbing silencer construction is suggested to use for noise reduction in large ducts by active absorbing silencers construction consisted of cascaded autonomous cassettes.

These examples are showing high possibilities and efficiency of active noise and vibration control method. From the other hand, it should be mentioned that ANVC systems have some drawbacks. E.g. in the case of it practical usage the problem of protection from the influence of interferences is arise, such as physical characteristics of operating medium (temperature, pressure, moisture, vibrations, dust etc.) which may influence significantly on the efficiency, reliability and 
durability of active system operation. Therefore, the problem of good protected active system construction design is arising. The next drawback is additional energy consumption etc.

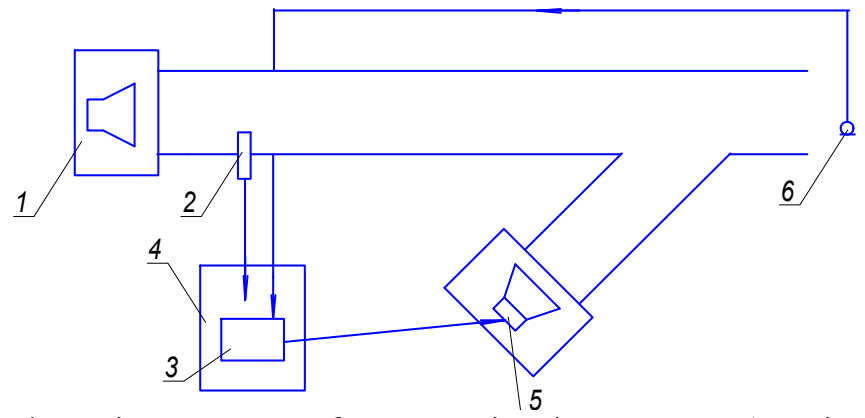

Fig. 1. Electronic ANC system of "Lotus Engineering" company. 1 - noise source;

2 - signal of determination of noise source; 3 - digital filter; 4 - computer;

5 - compensation source; 6 - feedback microphone

\section{Experience and perspectives of low frequency noise and vibration reduction generated by power plants gas guide systems by using of active noise and vibration control systems}

Some constructive schemes and creative ideas of ANVC systems in ducts are shown in papers $[7,9,12-15]$. Let us illustrate some examples.

The first example is reduction of automobile ICE intake low frequency noise by the active system with increased protection $[12,14]$. Intake system with active noise control unit includes inlet valve, throttle with disconnecting transmitter, air-cleaner chamber, air-cleaner intake pipe radiating noise to the field of compensation, etc. Receiver of sound pressure (microphone) is installed inside of air-cleaner chamber volume in the zone of low interferences and is connected with the element of adaptive control and amplifier. Active noise source (e.g. loudspeaker with moving magnet system and membrane) is connected with the field of compensation by means of the special pipe, frequency characteristic of which repeats the one of intake pipe. General construction of the system is shown in the Fig. 2. Inlet valve 1 generates oscillating air flow, which is transmitted through the air-cleaner chamber 2, intake pipe 4 and causes the sound radiation from the intake pipe open end 3 to the zone of compensation 5. Air flow oscillations in the chamber of air-cleaner are determined by microphone 8 and transmitting through to the amplifier 9 to the element of adaptive control 10 where active sound required phase characteristics are formed and amplitude amplification coefficient value is determined. Then the signal is amplified and transmitted to the loudspeaker 6 , membrane 7 of which radiates the sound through the special pipe into the field of compensation. Interference of intake noise, radiated by intake pipe open end, and the sound of compensation leads to undesired low frequency noise level attenuation.

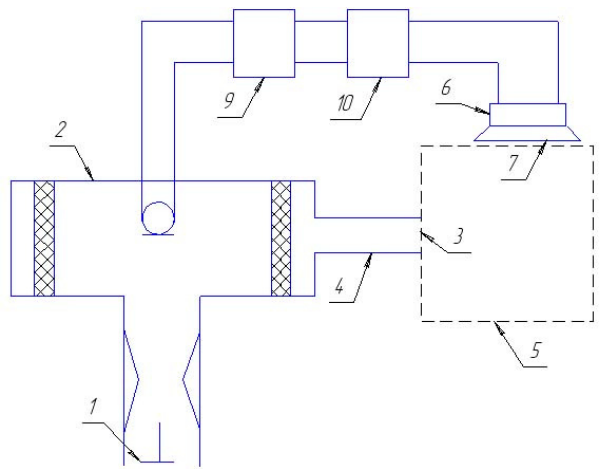

Fig. 2. Functional scheme of active ICE intake noise control system ([13]) 
The author's invention [14] is suggesting the construction of active noise control system allowing reduce noise from the several sources. Automobile internal combustion engine radiate both intake and exhaust noise. If reduced it separately by active mufflers strong coherent low-frequency sound radiation near to the air-suctioning pipe and exhaust pipe may appear. The essence of combined intake and exhaust noise compensation system (see Fig. 3) is that ICE 1 contains intake system 2, exhaust system 3 and the system of foaming of compensation signal 4 , including two-directional noise source 5 , radiating sound to acoustic waveguide 7 , connected with intake manifold 6; and to and acoustic waveguide 9, connected with exhaust pipe 8 . Noise source 5 is joint with control unit 10, connected with sensor 11 of ICE frequency of operation. Oscillations of loudspeaker membrane are assigned by control unit 10. Membrane radiated sound in opposite directions in anti-phase.

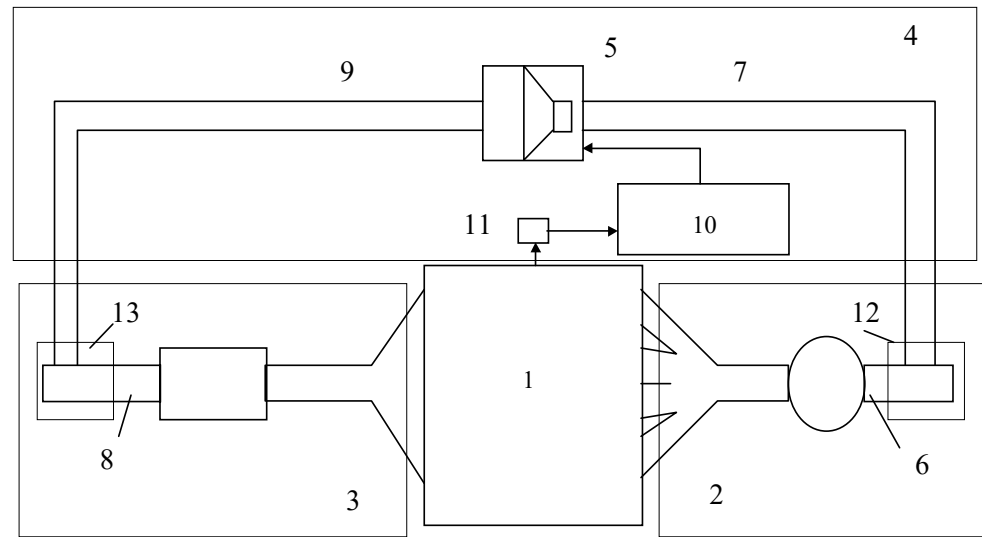

Fig. 3. Combined ICE intake and exhaust noise active muffler ([15])

The next example is development of active noise control system of automobile ICE intake suppressing maximal noise harmonics is described in papers [7, 13]. The forming of compensation signal is provided by means of sound generator control unit, which is analyzing the signal of sensing element. Sound compensating signal with necessary amplitude and phase is transmitted through the special pipe to the loudspeaker, which is radiating the sound signal directly to the zone of compensation. This zone dimensions are not exceed 1/12 value of primary sound source wavelength. Energy loss of this system will be minimized in view of the fact that sound signal levels in zone of compensation are significantly lower than sound signal levels of the system. The input of compensating signal to the zone of compensation depends on the throttle opening position. To minimize the energy consumption, we may use this system operation only in case of maximal or near to maximal angle of throttle opening. Amplitude and phase of active signal are selected by experiments for different engine operation conditions, than these data are used by signal generation control unit.

In practical usage of ANC systems, the problem of system elements protection from external interferences arises. External interferences may change the system frequency response, directional characteristics, impedance, sensitivity etc. Among of external interferences it is necessary to point out physical characteristics of operating medium (high pressure and velocity of gas flow, high temperature, moisture variations, dust or environmental dirties etc.); masking interferences of other noise and vibration sources; external vibration sources; affecting of ANC system elements by active chemical components in flow etc.

Further improvement of active noise control and vibration system elements protection allows the system to operate with higher efficiency and reliability. Widely, achieving good results in the solution of this problem helps us to extend the possibilities of active noise and vibration control systems practical application. Thus, it is necessary to develop ANVC systems with improved protection from external and internal factors impact, including ANVC systems elements 
protection, especially "weak links" - the elements of an active noise control system for ducts which are most sensitive to the influence of external factors. Usually such "weak links" are actuators (loudspeakers) and sensors (microphones).

The next tasks are reduction of energy consumption during ANVC systems operation (for example, by using of alternative sound energy sources and sensors), increasing of durability and reliability of ANVC systems, decreasing of the cost of ANVC systems etc.

\section{Conclusions}

Gas pressure pulsations (oscillations) in gas guide systems of power plants are generating intensive noise and vibration in low frequency range. Analysis of ANVC systems application for low frequency noise and vibration reduction in gas guide systems of power plants is described. Results of analysis of application of ANVC systems are showing high possibilities and efficiency of ANVC method. In a meantime, existing constructions of ANVC systems are having some drawbacks. E.g. in the case of it practical usage the problem of protection from the influence of interferences is arise, such as physical characteristics of operating medium (temperature, pressure, moisture, vibrations, dust etc.) which may influence significantly on the efficiency, reliability and durability of active system operation. Therefore, the problem of good protected active system construction design is arising. The next drawback is additional energy consumption, etc.

Author's experience of development of constructions and technical solutions of ANVC systems for cancellation of noise and vibration in low frequency range in power plants gas guide systems is presented. Some author's constructions are described.

Further perspectives of increasing of efficiency of ANVC control of low frequency noise and vibration reduction generated by power plants are discussed. It is necessary to develop ANVC systems with improved protection from external and internal factors impact, to reduce energy consumption during ANVC systems operation, to increase durability and reliability of ANVC systems, to decrease the cost of ANVC systems etc.

The results of research described in this paper may be useful for further improvement of active noise and vibration control systems constructions, will allow increase the efficiency of low frequency noise and vibration reduction in gas guide systems of power plants and to increase the period of ANVC systems application.

\section{Acknowledgement}

The author would like to express many thanks to Russian Scientific Foundation for financial supporting of research described in this paper by the grant, project number: 20-19-00222.

\section{References}

[1] Hansen C. H., Snyder S. D. Active Control of Noise and Vibration. Cambridge University Press, UK, 1997, p. 1267.

[2] Kobayashi Y., Fujioka H. Inexpensive implementation of active noise control systems for one-dimensional duct with application to a ventilating system. Proceedings of the 13th International Congress on Sound and Vibration, Austria, 2006.

[3] Krüger J., Leistner Ph. Calculation of actively absorbing duct linings. International Symposium on Active Control of Sound and Vibration, Hungary, 1997.

[4] McDonald A. M., Hutchins S. M., Renwick N., Stothers I., Brockie K. H. Attenuation of Dynamic Pressure Fluctuations in Ducts. International Patent Application WO 91/09214 A1, 1991.

[5] Mechel F. P. Hybrider Schalldämpfer. German Patent Application No. 4027 511 C1, 1991.

[6] Scheuren J. Engineering applications of active sound and vibration control. Noise Control Engineering Journal, Vol. 53, Issue 5, 2005, p. 197-210.

[7] Starobinski R. N., Vasilyev A. V., Gordeev V. N., Vasilyev V. V. The System of Active Suppression of Internal Combustion Engine Intake Noise. Patent of Russia No. 2096651, 1997. 
[8] Swinbanks M. A. The active control of sound propagating in long ducts. Journal of Sound and Vibration, Vol. 27, 1973, p. 411-436.

[9] Vassiliev A., Starobinsky R., Bakharev N. Low-frequency automobile intake noise reduction using active noise control system. 15th International Congress of Acoustics, Norway, 1995, p. 327-330.

[10] Winkler J., Elliott S. J. Adaptive control of broadband sound in ducts using a pair of loudspeakers. Acustica, Vol. 81, Issue 5, 1995, p. 475-488.

[11] Wise S., Leventhall G. Active noise control as a solution to low frequency noise problems journal of low frequency noise. Vibration and Active Control, Vol. 29, Issue 3, 2010, p. 119-137.

[12] Vassiliev A. V. Automobile engine low frequency noise reduction by complex using of active noise control method. International Conference on Noise and Vibration Engineering, Belgium, 2000, p. 37-44.

[13] Vassiliev A. V. Compact active noise control units for automobile intake low-frequency noise attenuation. International Symposium on Active Control of Sound and Vibration, Hungary, 1997, p. 587-594.

[14] Vasilyev A. V. Automobile internal combustion engine low frequency noise reduction using active noise control solutions. Journal "Akustika", Vol. 34, 2019, p. 113-117.

[15] Vasilyev A. V., Mokrinsky A. V. System of Internal Combustion Engine Intake and Exhaust Active Noise Suppression. Patent of Russian Federation for Invention No. 2240427, 2004. 